\title{
Electron transfer dynamics
}

\author{
JOSHUA JORTNER ${ }^{a *}$, M BIXON ${ }^{\mathrm{a}}$ and MARK A RATNER ${ }^{\mathrm{b}}$ \\ 2School of Chemistry, Tel Aviv University, Tel Aviv 69978, Israel \\ 'Department of Chemistry, Northwestern University, Evanston, Illinois 60208, \\ USA
}

\begin{abstract}
This paper surveys the current 'state-of-art' of the theoretical understanding of electron transfer dynamics in donor-acceptor systems, which provide the conceptual and technical basis for solar energy conversion via optical and optoelectronic molecular devices and for the primary charge separation in photosynthesis.
\end{abstract}

Keywords. Electron transfer dynamics; non adiabatic ET theory; intramolecular vibrational modes; vibrational relaxation.

\section{Introduction}

Energy storage and disposal via electron transfer (ET) in molecular, supermolecular and biophysical systems ${ }^{1,2}$ is expected to provide a central conceptual and technical basis for solar energy conversion via molecular optical and optoelectronic devices, and for the conversion of solar energy into chemical energy in the primary charge separation processes in photosynthesis. ET in supermolecules falls into two general categories

(1) Supermolecules ${ }^{3-6}$ consisting of an electron donor (D), and an electron acceptor (A), linked by a nonrigid or a rigid molecular bridge (B).

(2) Biophysical systems such as the photosynthetic reaction centers (RC) of bacteria and plants, ${ }^{7-10}$ where the primary process proceeds via a sequence of well organized, highly efficient, directional and specific ET processes between prosthetic groups embedded in the protein medium.

ET processes fall into two major types (i) ET in ground electronic states. Such processes are important for electronic transport in molecular materials, as well as for charge transfers in molecular devices. The latter can be induced by charge injection in external fields, by electrochemical processes or triggered by the capture of a solvated electron or by the formation of a precursor ion. (ii) ET in electronically-vibrationally excited states. These processes are triggered by optical excitation and may encompass also a variety of other intramolecular and intermolecular radiationless processes, e.g., electronic-vibrational relaxation, vibrational energy redistribution or medium-induced vibrational relaxation in conjunction with ET. These excited-state ET processes are significant for time-resolved triggering of charge transport and separation in molecular devices, e.g., molecular wires, switches and rectifiers ${ }^{11}$. These may consist of synthetic supermolecules ${ }^{11}$ or of components of biophysical systems, ${ }^{11}$ which may be realized in

\footnotetext{
* For correspondence
} 
artificial photosynthesis ${ }^{7-10}$. From the mechanistic point of view, photophysical and photobiological ET from an electronically excited donor proceeds via a direct process $D A \stackrel{h v}{\longrightarrow} D^{*} A \stackrel{E T}{\longrightarrow} D+A-$, a unistep superexchange mediated process $D B A \stackrel{h v}{\longrightarrow} D^{*} B A \stackrel{E T}{\longrightarrow}$ $D+B A-$ or a multistep sequential process $D B A \stackrel{h v}{\longrightarrow} D^{*} B A \longrightarrow D+B-A \rightarrow D+B A-$.

The control of ET in $D B A$ or $D A$ systems in solution, ${ }^{1}$ in a solid, ${ }^{2,12}$ in a protein ${ }^{7-10}$ or within an "isolated" solvent-free supermolecule ${ }^{13}$ can be accomplished by (1) Structural control. "Molecular engineering" of the $D, A$ and $B$ subunits determines the molecular energetics and the direct $D-A$ superexchange $D-B-A$ electronic coupling. (2) Intramolecular dynamic control of the nuclear equilibrium configurational changes (i.e. nuclear distortions) accompanying $\mathrm{ET}^{2,18}$ (3) Medium control of "conventional" ET in a solvent or in a cluster. The function of the medium on the $D B A \rightarrow D^{+} B A^{-}$is:. ${ }^{13}$ (i) The energetic stabilization of the ionic states, (ii) the coupling of the electronic states with the medium nuclear motion, which originates from short-range and long-range interactions in polar solvents, short-range interactions with $C-H$ group dipole in nonpolar hydrocarbons and with polar amino acid residues in proteins. (4) Dynamic medium control of $\mathrm{ET}^{14-17}$ involving: (i) the medium acting as a heat bath. The relaxation of the medium polar nuclear modes is often fast on the time scale of the electronic ET processes, which then constitute the rate determining step, (ii) dynamic solvation effects of $D B A$ or/and $D^{+} B A^{-}$determine the ET dynamics when condition (i) is violated and solvent controlled ET may be exhibited, (iii) specific dynamic control of pathways by solvent motions ("gating"), (iv) very slow solvent relaxations such as in glassy matrices, that lead to reduced solvent reorganization energies.

The structural, intramolecular, solvent and dynamic control of ET will allow for the design of molecular systems where ET is: (a) Ultrafast (on the time scale of $\sim 1$ psec $\sim 100 \mathrm{fsec}$ ), overwhelming any energy waste processes, (b) highly efficient, eliminating any back reactions, (c) stable with respect to the predictable variation of molecular and medium properties, (d) practically invariant with respect to temperature changes.

\section{Basic ET theory}

The nonadiabatic ET rate is given $b^{2,18}$

$$
k=(2 \pi / \hbar) V^{2} F,
$$

where $V$ is the electronic coupling and $F$ is the thermally averaged nuclear vibrational Franck-Condon factor. This microscopic description rests on the following description and conditions (a) ET is described as a radiationless transition. (b) The BornOppenheimer separability of electronic and nuclear motion applies, allowing description of the system in terms of diabatic potential surfaces (figure 1). (c) The electronic coupling is sufficiently weak to warrant the description of the radiationless transition in the nonadiabatic limit. (d) Microscopic ET rates are insensitive to medium dynamics. This state of affairs is realized under one of the following conditions, (i) The common situation of fast medium vibrational dynamics, which allows for the separation of time scales with the microscopic ET rate constants constituting the rate determining step, ${ }^{14}$ (ii) The microscopic ET rates weakly depend on the distribution in the initial $D^{*} B A$ vibronic manifold ${ }^{14}$.

The electronic coupling in the DA is determined by a direct exchange contribution $V_{D A}=\left(\phi_{D A}^{0}|\hat{H}| \phi_{D^{-} A^{-}}^{0}\right)$. In the bridged DBA system the electron can be described as 


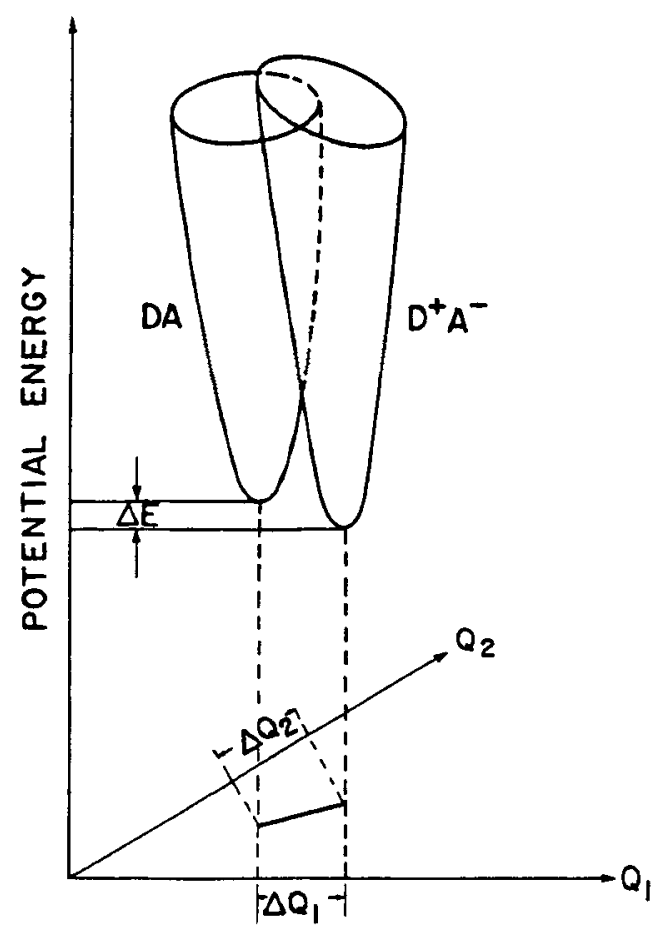

Figure 1. Diabatic nuclear potential energy surfaces for $D A \rightarrow D^{+} A^{-} E T$. The nuclear coordinates incorporate the medium and the intramolecular coordinates. The energy gap is $\Delta E$, while the displacement between the equilibrium configurations is marked as $\Delta Q_{1}$.

follows, (i) For resonance coupling, i.e., $\Delta G_{D B}<0$ (where $\Delta G_{D B}$ is the energy gap between $D B A$ and $D^{+} B^{-} A$ ), the process is sequential with the first step being given by exchange $V_{D B}=\left(\phi_{D B A}^{0}|A| \phi_{D^{+} B^{-} A}^{0}\right)$ between the electronic states of DBA and $\mathrm{D}^{+} \mathrm{B}^{-} \mathrm{A}$. (ii) For off-resonance coupling, i.e., $\Delta G_{D B} \gg 0, V$ involves superexchange coupling $V_{\text {super }}=V_{D B} V_{B A} / \delta E_{B}$, where $\delta E_{B}=\left(\Delta G_{D B}+\lambda_{D B}\right)$ is the vertical energy difference between the potential energy surfaces of $D B A$ and $D^{+} B^{-} A$, and $\lambda_{D B}$ is the reorganization energy between these states. For superexchange between $D, B$ and $A$ systems, both direct and superexchange interactions require the evaluation of individual pair $V_{D A}, V_{D B}$ and $V_{B A}$ couplings ${ }^{19-22}$. In both cases many-electron computations, transcending the naive one-electron picture, have to be invoked. The accumulated information concerning the distance dependence of both direct and superexchange interactions is that both interactions are expected to exhibit an exponential distance dependence $e^{18,2-6,23}$

$$
V=a \exp \left(-\beta R_{\mathrm{DA}}\right)
$$

where $R_{D A}$ is the (either edge-to-edge or center-to-center) $D-A$ distance. The distance dependence of intramolecular superexchange interactions in synthetic supermolecules and in the photosynthetic reaction center (figure 2) was inferred from the analysis of either ET rates or optical charge transfer spectra. The general trend confirms the 


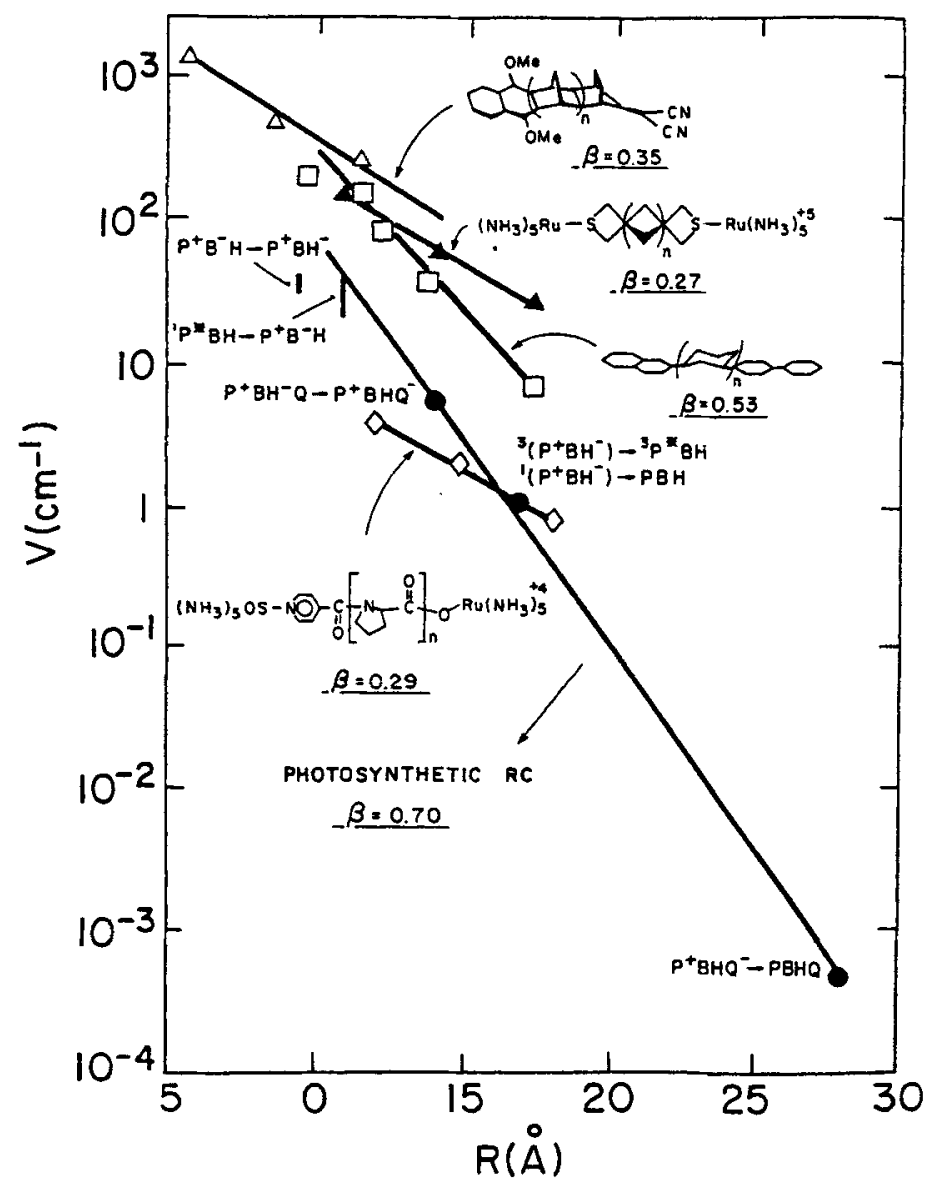

Figure 2 The distance dependence of $V$ for synthetic supermolecules obtained from kinetic and spectroscopic data (references 3-6) and for the bacterial photosynthetic RC obtained from analysis of kinetic data (references 7-10). The system specific $\beta$ values of equation 2 (in $\AA^{-1}$ ) are presented on the figure.

exponential relation, (2), quantified by $\beta$. The exponent $\beta$ is system specific, so that the exponential distance dependence of $V\left(\right.$ and of $k \propto V^{2} \propto \exp \left(-2 \beta R_{D A}\right)$ ) is not universal. On the basis of these exponential relations one cannot infer, apriori, whether the electronic coupling is direct or superexchange mediated, and further theoretical input is required. The minimization of $\beta$ will be desirable for molecular wires. It has been proposed (as follows from the simple perturbation theory) that $\beta$ can be minimized by reducing the gap between the state with the electron localized on $D$ or $A$, and that with the electron present on $B .{ }^{24}$ Indeed, if this gap vanishes, resonant tunneling is expected, as seen in the inelastic electron tunneling spectroscopy ${ }^{25}$ and in recent LangmuirBlodgett measurements, ${ }^{26,27}$ as well as inferred from simple model computations. ${ }^{28-31}$ In these truly resonant situations the exponential decay, (2), is not expected. In conductive polymers such as doped $(\mathrm{CH})_{x}$, essentially resonant transfers (vibronically mediated) occur, and equation 2 is not exhibited. 
The nuclear Franck-Condon factor $F$ in equation 1 contains the thermal average of the overlap integrals between the initial and final vibrational states of the system, under the restriction of energy conservation. For two harmonic potential energy surfaces characterized by identical reduced masses $\left\{\mu_{i}\right\}$ frequencies $\left\{\omega_{I}\right\}$ and normal modes $\left\{Q_{i}\right\}$, with nuclear displacements $\left\{\Delta Q_{i}\right\}$ of the equilibrium coordinates (figure 1), $F$ takes the form of the Fourier transform ${ }^{18}$

$$
F=(2 \pi \hbar)^{-1} \exp (-G) \int_{-\infty}^{\infty} \mathrm{d} t \exp (i \Delta E t / \hbar) \exp \left[G_{+}(t)+G_{-}(t)\right]
$$

where $\Delta E$ is the energy gap, and

$$
\begin{aligned}
& G_{ \pm}(t)=\sum_{i}\left(\Delta_{i}^{2} / 2\right)\left\{\frac{\bar{v}_{i}+1}{v_{i}}\right\}^{1 / 2} \exp \left( \pm i \omega_{i} t\right), \\
& G=G_{+}(0)+G_{-}(0) \\
& \bar{v}_{i}=\left[\exp \left(h \omega_{i} / k_{B} T\right)-1\right]^{-1}, \\
& \Delta_{i}=\left(\mu_{i} \omega_{i} / h\right)^{1 / 2} \Delta Q_{i},
\end{aligned}
$$

$\bar{v}_{i}$ denotes the thermal average and $\Delta_{I}$ is the reduced displacement of the ith mode, while $\exp (-G)$ is the Debye-Waller factor. Equation 3 was advanced for multiphonon optical transitions, for radiationless transitions in general and for ET in particular.

The vibrational modes incorporated in equation 3 include, (i) Low-frequency medium modes, often approximated by a single frequency $\omega_{m}$ with an effective $\Delta_{m}$. "Intelligent guesses" for glasses result in $\omega_{m} \approx 10-100 \mathrm{~cm}^{-1}$. It seems that for a polar solvent a phonon picture is not applicable, while for a protein medium ${ }^{32,33} \omega_{m} \approx 100 \mathrm{~cm}^{-1}$. The medium reorganization energy is $\lambda_{m}=S_{m} h \omega_{m}$, where $S_{m}=\Delta_{m}^{2} / 2$. For polar solvents $\lambda_{m} \approx 4000-8000 \mathrm{~cm}^{-1}$ while for the protein RC $\lambda_{m} \approx 800-3000 \mathrm{~cm}^{-1}$, (ii) intramolecular modes in the range $\omega \approx 100-3000 \mathrm{~cm}^{-1}$. A complete treatment of the ET requires the incorporation of all the coupled (quantum) intramolecular modes, providing the basis for intramolecular ET dynamics. ${ }^{13,18}$ Often, but not exclusively, the intramolecular modes can be approximated by a single molecular frequency $\omega_{c} \approx 1000-1500 \mathrm{~cm}^{-1}$ and a dimensionless shift $S_{c}=\Delta_{c}^{2} / 2 \approx 0.5-1 \cdot 0 .{ }^{32}$

Energy gap laws constitute a major generalization of microscopic relaxation phenomena, which originate from the dependence of $\mathrm{F}$, (3), on $\Delta E$ (or $\Delta G$ ). The quantum nature of the intramolecular vibrational modes is usually prevalent at all the relevant temperatures, as $k_{B} T \ll \hbar \omega_{m},(3)$, reduces to ${ }^{18}$

$$
F=\left(4 \pi \lambda_{m} k_{B} T\right)^{-1 / 2} \exp \left(-S_{c}\right) \sum_{n=0}^{\infty} \frac{S_{c}^{n}}{n !} \exp \left[-\frac{\left(\Delta E+\lambda_{m}+n \hbar \omega_{c}\right)^{2}}{4 \lambda_{m} k_{B} T}\right],
$$

providing a useful contribution for the calculation of nuclear contributions to the ET rate over a broad temperature domain. In the limit $S_{c}=0$, equation 8 reduced to the classical Marcus relation ${ }^{2}, F_{c l}\left(S_{c}=0\right)=\left(4 \pi \lambda_{m} k_{B} T\right)^{-1 / 2} \exp \left[-\left(\Delta E+\lambda_{m}\right)^{2} / 4 \lambda_{m} k_{B} T\right]$ with a Gaussian activation energy. The (free) energy dependence of $F$ (figure 3 ) provides a demonstration of the classical Marcus relation in the normal region $\left(-\Delta E \leqslant \lambda_{m}\right)$ and in the activationless domain $\left(-\Delta E=\lambda_{m}\right)$, while in the inverted region $\left(-\Delta E \geqslant \lambda_{m}\right)$ marked deviations from the classical relation are exhibited (i.e., $F>F\left(S_{c}=0\right)$ ) due to the vibrational excitation of the intramolecular quantum modes of $D$ and $A$, accompanying ET. Quantum effects in the inverted region include a marked enhancement of 


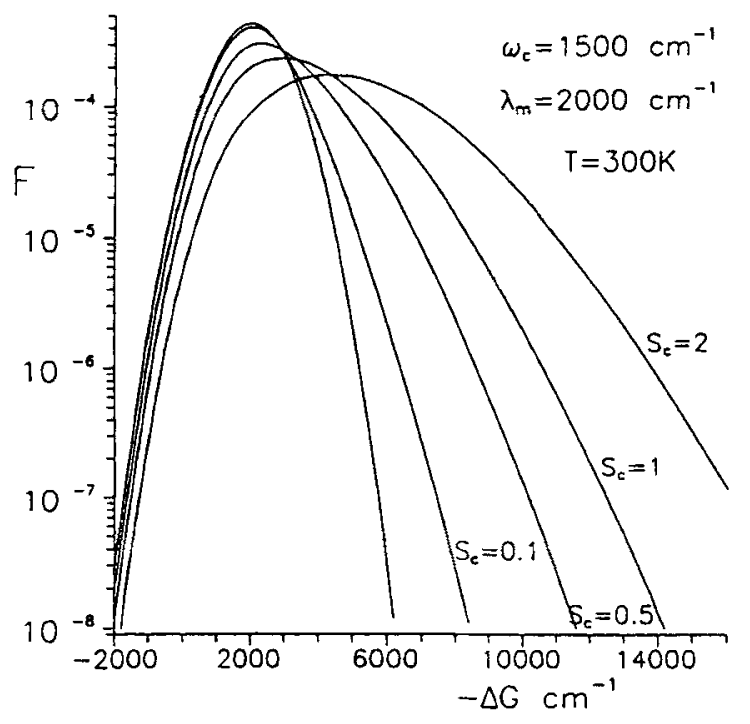

Figure 3. The energy gap dependence of the nuclear Franck-Condon factc:which incorporates the role of high frequency intramolecular mode(s).

the ET rate at constant $\Delta G$ (figure 3) and a surprisingly weak temperature dependence of $k$ for strongly exoergic reactions. ${ }^{32}$ The contribution of high-frequency intramolectlar modes to ET dynamics opens up the possibility of mode-specific ET. Provided that vibrational relaxation of a high-frequency mode is slow on the time scale of ET (involving an equilibrated manifold of all the other medium and intermolecular modes), nonadiabatic ET from distinct photoselected vibrational states will be stat: specific, as demonstrated by Spears ${ }^{34,35}$ for the ET process $\left[\mathrm{Co}(\mathrm{Cp})_{2}\right]\left[\mathrm{Co}(\mathrm{CO})_{4}\right]-$ $\left[\left(\mathrm{Co}(\mathrm{Cp})_{2}\right]^{+}\left[\mathrm{Co}(\mathrm{CO})_{4}\right]^{-}\right.$from vibrationally excited high-frequency $\mathrm{CO}$ modes $\left(\omega_{c} \approx 2000 \mathrm{~cm}^{-1}\right)$.

The energy gap dependence of $F$ (figure 3 ) implies an optimization principle fer activationless ET. The $F$ factor is maximized $\left(F=F_{\max }\right)$ for $-\Delta E=\lambda_{m}+n h \omega_{c}$, with the dominating contribution to the sum in equation 8 originating from the $n$th term, so thas $F_{\max } \approx\left(4 \pi \lambda_{m} k_{B} T\right)^{-1 / 2}\left(S_{c}^{n} / n !\right) \exp \left(-S_{c}\right)$. This situation corresponds to activationless ET (i.e., for the lowest intersection of the multidimensional nuclear potential surfaces occurring at the minimum of the $D^{*} A$ or $D^{*} B A$ state) with a weakly temperature dependent $k \propto T^{-2:}$ non-Arrhenius-type rate. For typical values of $S_{c}=1$ the activationless ET is realized wit $F_{\max } \approx\left(4 \pi_{m} k_{B} T\right)^{-1 / 2} \exp \left(-S_{c}\right.$. The maximization of the Franck-Condon factor involves the optimization of the nuclear contribution to the ET rate.

In the nonadiabatic limit the optimal activationless rate is $k=\left(2 \pi V^{2} / h\right) F_{\mathrm{MAX}}$. For characteristic values $\lambda_{m}=4000 \mathrm{~cm}^{-1}$ for ET in polar solvents or $\lambda_{m}=1000 \mathrm{~cm}^{-1}$ in protein RC together with $S_{c} \approx 1$, we estimate (at room temperature) the activationless ET rate,

$$
\left(k / \mathrm{sec}^{-1}\right) \approx(1 \cdot 4-2 \cdot 7) \times 10^{8}\left(\mathrm{~V} / \mathrm{cm}^{-1}\right)^{2} .
$$

For typical $V$ values (figure 2), we estimate $k \approx 2 \times 10^{10} \mathrm{sec}^{-1}$ for $V=10 \mathrm{~cm}^{-1}$ and $V \approx 2 \times 10^{12} \mathrm{sec}^{-1}$ for $V \approx 100 \mathrm{~cm}^{-1}$. The upper limit of the maximized activationless 
ET rate is determined by the breakdown of the nonadiabatic formalism, which requires the extension of the nonadiabatic theory.

\section{Extensions of nonadiabatic ET theory}

(3a) Adiabatic ET. In the preceding section we have spelt out the conditions for the applicability of the nonadiabatic ET theory. Condition $(\mathrm{C})$ for weak electronic coupling implies that the Landau-Zener parameter is small, i.e., $\gamma \approx 2 V^{2} / \hbar \omega_{m}\left(\lambda_{m} \hbar \omega_{m}\right)^{1 / 2}<1$, where $\omega_{m}$ is an effective medium frequency. ${ }^{36,37}$ Taking characteristic values of $h \omega_{m}=100 \mathrm{~cm}^{-1}$ both for a polar solvent and for a protein medium, nonadiabatic ET prevails for $V \leqslant 200 \mathrm{~cm}^{-1}$ in a polar solvent $\left(\lambda_{m} \approx 4000 \mathrm{~cm}^{-1}\right)$ and for $V \leqslant 100 \mathrm{~cm}^{-1}$ in a protein medium $\left(\lambda_{m} \approx 1000 \mathrm{~cm}^{-1}\right)$. In figure 4 we have schematically marked the upper limit for the $y$ values, which still correspond to nonadiabatic ET. When the electronic coupling is sufficiently strong, i.e., $\gamma>1$, the adiabatic limit for ET applies. The ET rate is then given by the Holstein formula,

$$
K=\left(\omega_{m} / 2 \pi\right) \exp \left(-E_{a} / k_{B} T\right) .
$$

For activationless ET, the adiabatic rate constant is $k \approx \omega_{m} / 2 \pi$. These considerations are limited to those systems where the contribution of the medium modes dominates. In the systems where ET is dominated by high frequency intramolecular modes, the description of nonadiabatic ET should be replaced by the theory of intramolecular radiationless transitions. ${ }^{13}$

(3b) Solvent-controlled ET. The breakdown of assumption (D) implies that solvent relaxation, rather than the microscopic electronic processes, constitutes the rate determining step for ET. For ET in a system solely characterized by coupling to the medium $\left(S_{c}=0\right)$, which corresponds to the normal Marcus region $\left(-\Delta E \leqslant \lambda_{m}\right)$, the realization of solvent-controlled ET is determined by the magnitude of the solvent adiabaticity parameter ${ }^{14} \kappa=4 \pi V^{2}\langle\tau\rangle / \hbar \lambda_{m}$, where $\langle\tau\rangle$ is the longitudinal dielectric relaxation time $\tau_{L}$, e.g., $\tau_{L}=200 \mathrm{fsec}$ for water, $\tau_{L}=190 \mathrm{fsec}$ for acetonitrile and $\tau_{L}=1.2$ psec for methyl acetate at room temperature. ${ }^{14}$ For the membrane protein
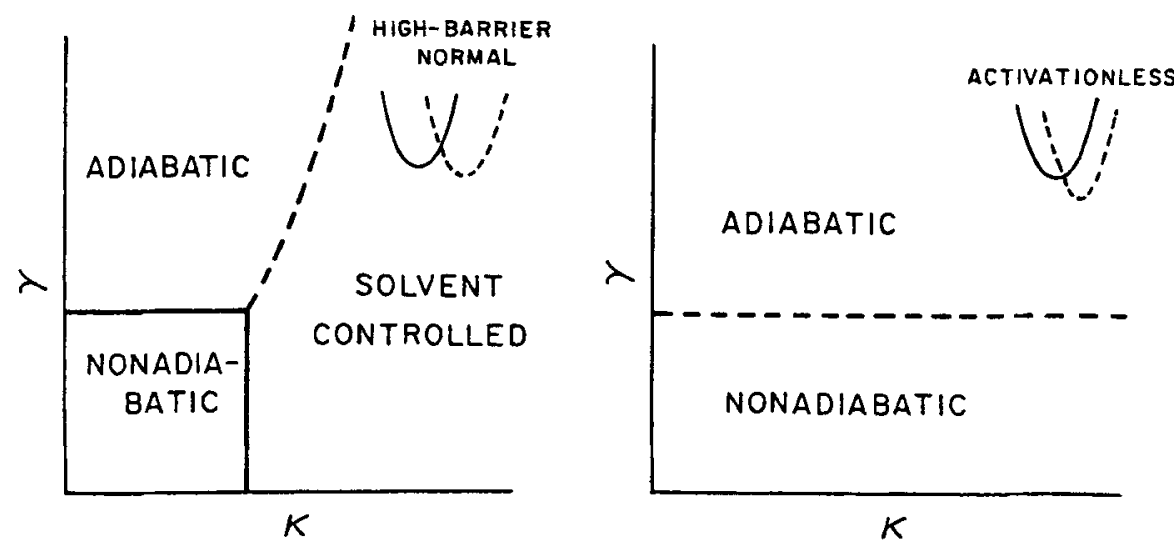

Figure 4. Domains for ET for the normal ET and for the activationless case. 
medium of the $\mathrm{RC},{ }^{7-11,38,39}$ molecular dynamics simulations give $\langle\tau\rangle=100$ fsec over the temperature domain $10 \mathrm{~K}-300 \mathrm{~K}$. The medium controlled ET rate is given by ${ }^{14}$ $k=k^{N A} /(1+\kappa)$, where the nonadiabatic rate $k^{N A}$ is given by equation 1 . In the limit $\kappa \gg 1, \propto k^{N A} / \kappa \propto\langle\tau\rangle^{-1}$, being independent of $V$ (figure 4).

Is this formalism relevant for activationless ET? Medium controlled activationless ET will be manifested only provided that the microscopic rates are sensitive to the details of the distribution of the initial states. For activationless ET both model calculations in the classical limit and numerical computations reveal that the microscopic ET rates $k_{i}$ are quite insensitive to the initial vibrational state. ${ }^{41}$ This weak excess energy dependence of $k_{i}$ is compatible with the weak temperature dependence of activationless ET. Accordingly, activationless ET is invariant with respect to medium relaxation dynamics, with the rate being independent of $\kappa$ (figure 4). This analysis provides a possible explanation for the recent experimental observations of some ET rates, which substantially exceed the $\tau_{L}^{-1}$ limit predicted for solvent controlled ET. Kobayashi et al ${ }^{42}$ reported ultrafast ET rates in (Nile blue ${ }^{+}$) (TMPD) with $k \approx(100 \mathrm{fsec})^{-1}$ which corresponds to $k \approx 50 / \tau_{L}$, while Heitele et a ${ }^{43}$ observed for ET in porphyrin-quinone cyclophanes independence of $k(\approx 1 \mathrm{psec})^{-1}$ on $\kappa(=0 \cdot 1-400)$ over a broad region. ET in both systems seems to correspond to activationless ET (or inverted region) ${ }^{41}$. The primary ET reactions in the photosynthetic RC are nearly activationless, ${ }^{7-10}$ so that the medium dynamics (with $\langle\tau\rangle \approx 100 \mathrm{fsec}^{40}$ are not manifest explicity in the rates. ${ }^{38,39,41}$ Thus activationless ET can be appreciably faster than previously expected, and is not limited by solvent dynamics.

\section{An upper limit for activationless ET rates}

For activationless ET the nonadiabatic limit is expected to prevail, being characterized by $k \propto V^{2}$ with increasing $V$, until the adiabatic limit for ET will be achieved constituting an upper limit for the rate. An approximate estimate for the upper limit for the activationless ET rate in a system where ET is dominated by coupling with medium modes can be inferred from (10) in conjunction with the Landau-Zener parameter $\gamma \leqslant 1$. For a typical maximal value of medium modes of $\omega_{M} \approx 30-100 \mathrm{~cm}^{-1}$, one estimates $\left(\omega_{M}\right) \sim(1000 \mathrm{fs})^{-1}-(300 \mathrm{fs})^{-1}$ for the upper limit for the rate. In a realistic system where the coupling to an intramolecular high-frequency mode(s) $\omega_{c}$ is important, the upper limit for the ET rate will be determined by this vibrational frequency. Thus for $\omega_{c} \cong 300-1000 \mathrm{~cm}^{-1}$ the upper limit for the rate is $\sim(30 \mathrm{fs})^{-1}-(100 \mathrm{fs})^{-1}$. The fastest room temperature ET rates are recorded to date in synthetic supermolecules $k \approx(100 \mathrm{fs})^{-1}{ }^{40}$. In the $\mathrm{RC}$, with the rates for primary charge separation ${ }^{1} P^{+} B H \stackrel{k_{1}}{\longrightarrow} P^{+} B^{-} H \stackrel{k_{2}}{\longrightarrow} P^{+} B H^{-}$being $k_{1} \approx(3000 \mathrm{fs})^{-1}$ and $k_{2} \approx(1000 \mathrm{fs})^{-1}$ at $T=300 \mathrm{~K},{ }^{44-47} k_{1}$ and $k_{2}$ are still lower than maximal ET rates in a system where the coupling to a $\omega \sim 100 \mathrm{~cm}^{-1}$ frequency (i.e., the bacteriochlorophyll dimer frequency) dominates the ET dynamics.

The pertinent time scales for ET can be inferred by drawing the analogy between these processes and intramolecular and condensed phase radiationless transitions. The relevant physical parameters are the (pure) electronic coupling $V$, the (average) Franck-Condon factors (FC), the medium-induced vibronic density of states $\rho$, the medium-induced vibrational relaxation rate $\Gamma=\hbar / \tau_{V R}$ (where $\tau_{V R}$ is the vibrational relaxation time) and the characteristic medium frequency $\omega_{m}$. The condition for strong 
interstate coupling, i.e., $V(F C)^{1 / 2} \rho \gg 1$, implies that the time scale for nonadiabatic ET $\tau_{E T}=\left[(2 \pi / h) V^{2}(F C) \rho\right]^{-1}$ is longer than the mixing lifetime $\tau_{\mathrm{MIX}}=\left[V(F C)^{1 / 2} / h\right]^{-1}$. In turn, for conventional nonadiabatic ET the separation of time scales implies that $\tau_{V R} \ll \tau_{E T}$. Finally, $\tau_{V R}$ cannot be faster than the period of vibrational motion, i.e., $\tau_{V R} \geqslant \omega_{m}$. The concept of the medium-induced vibrational relaxation time $\tau_{V R}$ of the internal vibrational states of the $D$ and $A$ centers has to be extended to incorporate also the solvent relaxation time $\langle\tau\rangle$ induced by the charge distribution. The description of solvent relaxation should also include short-time, short-range inertial effects. Thus for conventional nonadiabatic ET occurring from a thermally equilibrated manifold,

$$
\tau_{E T} \gg \tau_{M I X} ; \quad \tau_{E T} \gg \tau_{V R} \geqslant 1 / \omega_{m} ; \quad \tau_{E T} \gg\langle\tau\rangle .
$$

The upper limit for the time scale of ET dynamics is provided by the time scale of nuclear motion $1 / \omega_{m}$, which constitutes the adiabatic limit for activationless ET according to equation 10.

For ultrafast ET, where $\tau_{E T}$ is comparable to or smaller than $\tau_{V R}$ and/or $\langle\tau\rangle$, nonadiabatic ET occurs from a nonequilibrated vibronic (and solvent) manifold. The ET dynamics is determined by the excess vibrational energy dependence of the microscopic interstate nonadiabatic ET rates, and by vibrational and solvent intrastate vibrational energy relaxation rates. With the advent of femtosecond lasers, the dynamics of coherent vibrational wavepackets of $D^{*} A$ (or $D^{*} B A$ ) states become amenable to experimental interrogation. ${ }^{42,48,49,50}$ The dynamics involves the interplay between intrastate vibrational relaxation, interstate electron transfer and dephasing of the vibrational wavepacket. In addition to the level depletion time scales $\tau_{E T}$ and $\tau_{V R}$, the pure vibrational dephasing time scale $\tau_{p} \sim 100-1000$ fsec in solution ${ }^{48-51}$ and in biophysical system ${ }^{47}$ enters. Exploration of the response of molecular electronic systems to femtosecond laser excitation will provide a new approach for the exploration of ET dynamics on the time scale of nuclear motion, attaining selectivity, sensitivity and a high yield of basic ET processes.

\section{Vibrational coherence in electron transfer}

The experimental observation of vibrational coherence effects for reactants and products in nonadiabatic ET dynamic ${ }^{48-51}$ falls in the category of a wealth of electronic and vibrational coherence effects induced by femtosecond laser excitation in the condensed phase. ${ }^{48,51-54}$ These vibrational coherence effects originate from the time evolution of wavepackets of nuclear states, which is manifested by oscillatory temporal evolution, i.e., quantum beats, with the characteristic frequencies corresponding to the energy differences between the coherently excited nuclear (or electronic-nuclear) states. Coherent ET dynamics ${ }^{48,51,55}$ transcendents the description of nonadiabatic dynamics in terms of the rates given by (1). In particular, the description of vibrational coherence in nonadiabatic ET dynamics requires the introduction of the correlation parameters $^{55}$

$$
\left.\eta_{s s},=\left\langle V_{s \alpha} V_{\alpha s}\right\rangle\right\rangle /\left[\left\langle V_{s a}^{2}\right\rangle\left\langle V_{s \alpha}^{3}\right\rangle\right]^{1 / 2},
$$

where the matrix elements of the Hamiltonian $\underset{\sim}{(H)})$, i.e., $V_{s \alpha}=\langle s|\underset{\approx}{H}| \alpha\rangle$, correspond to the coupling of the (reactant) doorway states $|S\rangle,\left|S^{\prime}\right\rangle,\left|S^{\prime \prime}\right\rangle$ (characterized by the energies $\left.E_{s}, E_{s^{\prime}}, E_{s^{\prime}}\right)$ with the (product) quasicontinuum states $\{|\alpha\rangle\}$. \langle\rangle 
in (11) denotes averaged products over the energy range which includes $E_{s}$ and $E_{s}$. An analysis ${ }^{55}$ of the time evolution of an initially excited coherent wavepacket of doorway states,

$$
\Psi(0)=\sum_{s} A_{s}(0)|S\rangle
$$

where $A_{s}(0)$ are the excitation amplitudes, reveals that the interstate nonadiabatic dynamics is determined by the two sets of attributes,

(i) The microscopic rates $\left\{k_{s}\right\}$ from individual doorway states

$$
k_{s}=(2 \pi / \hbar) V^{2} \sum_{\alpha}|\langle s \mid \alpha\rangle|^{2} \delta\left(E_{s}-E_{\alpha}\right),
$$

(ii) The off-diagonal matrix elements of the decay matrix

$$
\Gamma_{s s}=(\hbar / 2) \eta_{s s}\left(k_{s} k_{s}\right)^{1 / 2}
$$

with $\eta_{s s}$ being determined by (11). The time dependence of the nonradiative probability $P(t)$ (for the relevant limit $\Gamma_{s s}\left\langle\left|E_{s}-E_{s}\right|\right)$ is

$$
\begin{aligned}
P(t)= & \sum_{s}\left|A_{s}(0)\right|^{2} \exp \left(-k_{s} t\right)+\sum_{s \neq s} A_{s}^{*}(0) A_{s}(0) \otimes \\
& \left.\otimes\left[\frac{i \Gamma_{s s}}{\left(E_{s}-E_{s}\right)}\right] \exp \left[i\left(E_{s}-E_{s}\right) t / \hbar\right] \exp \left[-k_{s}+k_{s}\right) / 2\right] .
\end{aligned}
$$

In (15) the first term corresponds to direct decay, while the second term represents quantum beats. The quantum beats terms in $P(t)$ are determined by the spectroscopic, energetic and dynamics properties of the doorway states which pertain to, (i) large preparation amplitudes $\left\{A_{s}(0)\right\}$, (ii) periods $T_{p}=h /\left|E_{s}-E_{s}\right|$ of quantum beats, (iii) modulation amplitudes determined by $\Gamma_{s s} /\left|E_{s}-E_{s}\right|$ and (iv) sufficiently large correlation parameters $\eta_{s s}$. While features (i) and (ii) provide the signature of the laser excitation conditions, features (iii) and (iv) constitute the characteristics of nonadiabatic nonradiative coupling and dynamics, providing the distinction between the experimental aspects of wavepacket preparation and the intrinsic manifestations of interstate dynamics. The ubiquity of vibrational coherence effects raises the conceptual question of the distinction between the experimental conditions of preparation and interrogation and the intrinsic aspects of relaxation and dephasing dynamics. Addressing the issue of how coherent excitation of a wavepacket of doorway states modifies nonadiabatic dynamics, we assert that indeed the temporal modulation amplitudes in $P(t)$ are determined by condensed phase dynamic parameters $\eta_{s s}$, however, the overall influence on the modulation of $P(t)$ is small (figure 5). On the other hand, the amplitudes of the pronounced quantum beats in the photon counting rate $I(t)$ from the excited wavepacket do not provide information on the nonradiative interstate dynamics, just reflecting radiative interference effects. ${ }^{55}$ Vibrational coherence effects in the electronically excited bacteriochlorophyll dimer $\left({ }^{1} P^{*}\right)$ of the bacterial photosynthetic reaction center $(R C)^{53}$ interrogated by the (spontaneous and induced) fluorescence decay $I(t)$ just provide spectroscopic information on features (i) and (ii) above, but not on the charge separation dynamics. 


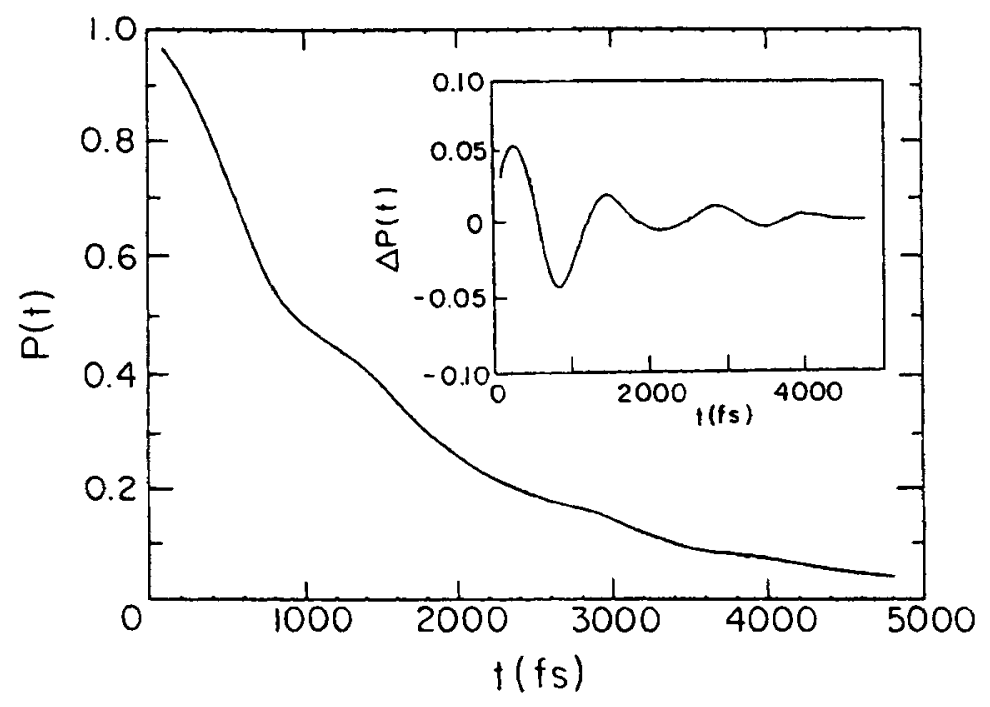

Figure 5. Temporal vibrational coherence in nonadiabatic dynamics, showing the nonradiative decay probability $P(t)$ of the reactants' manifold to a vibronic quasicontinuum. Data for a four-mode Franck-Condon system with frequencies $\omega / \mathrm{cm}^{-1}=(117,75,35,27)$, coupling parameters $S=(1 \cdot 0,1 \cdot 1,1 \cdot 2,3 \cdot 0)$, energy gap $\Delta E=500 \mathrm{~cm}^{-1}$ and electronic coupling $V=20 \mathrm{~cm}^{-1}$. The initial wavepacket consists of the seven lowest states in the doorway manifold with the amplitudes given by the appropriate vibrational overlap integrals from the ground electronic-vibrational state. The inset shows the time dependence of $\Delta P(t)=P(t)-\operatorname{Av}[P(t)]$, reflecting low amplitudes of the quantum beats.

\section{Perspectives}

Modern developments in the experimental and theoretical realms of ET dynamics raise some novel and interesting issues, which are being currently vigorously pursued. (i) Control and optimization of ET dynamics. (ii) Mode selective ET from state-selected intramolecular vibrational levels. (iii) Solvent dynamic control of ET in the context of the relation between ET and intramolecular radiationless transitions. (iv) Records for the attainment of the fastest time scales for ET. (v) Vibrational coherence effects on nonadiabatic ET providing new dynamic information (vi) The extension of ET concepts to a variety of problems in material and interface science, e.g., charge injection from electrodes, conductive polymers, STM imaging and molecular electronic responses. (vii) The primary processes of charge separation in photosynthesis, in the context of mechanisms and symmetry breaking of primary ET. ${ }^{7-10}$ These central issues pertain to structure-dynamics relations in biophysical and chemical dynamics.

The advent of femtosecond dynamics on the time scale of nuclear motion ${ }^{6}$ opened up new horizons in the exploration of ultrafast processes in general, and ET in chemical and biological systems in particular.

\section{Acknowledgements}

The support of the Volkswagen Foundation is gratefully acknowledged. 


\section{References}

1. Marcus R A 1964 Ann. Rev. Chem. 15, 155

2. Marcus R A and Sutin N 1985 Biochim. Biophys. Acta 811, 265

3. Closs G L, Calcaterra L T, Green N J, Penfield K W and Miller J R 1986 J. Phys. Chem. 90 , 3673

4. Stein C A, Lewis N A and Seitz G J 1982 J. Am. Chem. Soc. 104, 2596

5. Isied S S, Vassilian A, Wishart J F, Creutz C, Schwarz H A and Sutin N 1988 J. Am. Chem. Soc. 109, 635

6. Penfield K W, Miller J R, Paddon-Row M N, Cotsaris E, Oliver A M and Hush N S $1987 \mathrm{~J}$. Am. Chem. Soc. 109, 5061

7. Jortner J and Pullman B 1990 editors Perspectives in Photosynthesis (Dordrecht: Kluwer Academic Publishers)

8. Michel-Beyerle M E editor, The Reaction Center of Photosynthetic Bacteria (Springer Verlag, Berlin, 1990, 1995)

9. Deisenhoefer J and Norris J R editors, The Photosynthetic Reaction Center, Volumes I and II (Academic Press, New York, 1993)

10. Breton $\mathrm{J}$ and Vermeglio A editors, The Photosynthetic Reaction Center, Structure and Dynamics, Volumes I and II (Academic Press, New York, 1987, 1992)

11. Pecile C, Giacometti G and Bozio R 1993 editors, Proceedings 1st European Conference on Molecular Electronics. Mol. Cryst. Liq. Cryst. 234, 1-795

12. Mikkelsen K V and Ratner M A 1987 Chem. Rev. 87, 112

13. Bixon M and Jortner J 1993 J. Phys. Chem. 97, 13061

14. Rips I and Jortner J 1987 J. Chem. Phys. 87, 6513

15. Gaines G L, O'Neil M P, Svec W A, Niemczyk M P and Wasielewski M R 1991 J. Am. Chem. Soc. 113, 719

16. Hoffman B M and Ratner M A 1996 Inorg. Chim. Acta 243, 233

17. Sutin N, Brunschwig B, Johnson M K, King R B, Kutal D M, Kutrl C, Norton M C and Scott R A editors, Electron Transfer in Biology and the Solid State (ACS, Washington 1990) p. 65

18. Jortner J 1976 J. Chem. Phys. 64, 4860

19. Siddarth P and Marcus R A 1990 J. Phys. Chem. 94, 2985

20. Liang C and Newton M D 1993 J. Phys. Chem. 97,13083

21. Mikkelsen K V and Ratner M A 1989 J. Phys. Chem. 93, 1759

22. Shepard M J, Paddon-Row M N and Jordan K D 1993 Chem. Phys. 176, 289

23. Mujica V, Kemp M and Ratner M A 1994 J. Chem. Phys. 101, 6849, 6856

24. Joachim C and Vinussa J F 1996 Europhys. Lett. 33, 635

25. Hipps K W and Mazur U 1994 J. Phys. Chem. 98, 5824; Mazur U and Hipps K W 1994 J. Phys. Chem. 98, 8169

26. Martin A S, Sambles J R and Ashwell G J 1993 Phys. Rev. Lett. 70, 218

27. Fischer C M, Burghard M, Roth S and Klitzing K V 1994 Europhys. Lett. 28, 129

28. Mujica V, Kemp M, Roitberg A and Ratner M A 1996 J. Chem. Phys. 104, 7296. Coulomb staircases were treated earlier by Averin D V, Korotkov A N and Likharev K K 1991 Phys. Rev. B44, 6199

29. Sumetskii M 1993 Phys. Rev. B48, 4586; Sumetskii M 1991 J. Phys. Cond. Matt. 3, 2651; Sumetskii M 1985 Sov. Phys. JETP 62, 355

30. Landauer R 1957 IBM J. Res. Dev. 1, 223

31. Sautet $P$ and Joachim Ch. 1991 Chem. Phys. Lett. 185, 23

32. Bixon M and Jortner J 1991 J. Phys. Chem. 95, 1941

33. Todd M D, Nitzan A, Ratner M and Hupp J T 1994 J. Photochem. Photobiol. A82, 87

34. Spears K G, Wen X and Arrivo S 1994 J. Phys. Chem. 98, 9693

35. Sprears K G 1995 J. Phys. Chem. 99, 2469

36. Newton M D and Sutin N 1984 Ann. Rev. Phys. Chem. 35, 437

37. Jortner J and Bixon M 1993 Mol. Cryst. Liq. Cryst. 234, 29

38. Bixon M Jortner J and Michel-Beyerle M E 1991 Biophys. Biochim. Acta 1056. 31

39. Bixon M, Jortner J and Michel-Beyerle M E 1995 Chem. Phys. 197, 389

40. Schulten K and Tesch M 1991 Chem. Phys. 158, 421 
41. Bixon M and Jortner J 1993 Chem. Phys. 176, 467

42. Kobayashi T, Takagi Y, Kondori H, Kemnits K and Yoshihara K 1991 Chem. Phys. Lett. 180,416

43. Heitele H, Pöllinger F, Haberle T, Michel-Beyerle M E and Staab H 1994 J. Phys. Chem. 98 , 7402

44. Holzapfel W, Finkele U, Kaiser W, Oesterhelt D, Scheer H, Stilz H U and Zinth W 1990 Proc. Natl. Acad. Sci. USA 87, 5168

45. Chan C K, DiMango T M, Chen K X Q, Norris J and Fleming G R 1991 Proc. Natl. Acad. Sci. USA 88, 11202

46. Dressler K, Umlauf E, Schmidt S, Hamm P, Zinth W, Buchanan S and Michel H 1991 Chem. Phys. Lett. 193, 270

47. Vos M H, Rappaport F, Malbry J C, Breton J and Martin J L 1993 Nature 363, 320

48. Wynne K, Galli C and Hochstrasser R M 1994 J. Chem. Phys. 100, 4797

49. Reid P J, Silva C, Barbara P F, Karki L and Hupp J T 1995 J. Phys. Chem. 99, 2609

50. Arnett D C, Vohringer P and Scherer N F 1995 J. Am. Chem. Soc. 177, 12262

51. Wynne K, Reid G D and Hochstrasser R M 1996 J. Chem. Phys. 105, 2287

52. Bradforth S E, Jimenez R, Van Mourik F, Van Grondelle R and Fleming G R 1995 J. Phys. Chem. 99, 16179

53. Vos M H, Rappaport F, Lambry J C, Breton J and Martin J L 1993 Nature 36, 320

54. Chachisvilis M, Pullerits T, Jones M R, Hunger C N and Sundström V 1994 Chem. Phys. Lett. 224, 345

55. Bixon M and Jortner J Vibrational Coherence in Nonadiabatic Dynamics. J. Chem. Phys. (submitted) 